\title{
Electromagnetic Casimir effect for conducting plates in de Sitter spacetime
}

\author{
A. S. Kotanjyan, A. A. Saharian† H. A. Nersisyan \\ Department of Physics, Yerevan State University, \\ 1 Alex Manoogian Street, 0025 Yerevan, Armenia
}

March 8, 2018

\begin{abstract}
Two-point functions, the mean field squared and the vacuum expectation value (VEV) of the energy-momentum tensor are investigated for the electromagnetic field in the geometry of parallel plates on background of $(D+1)$-dimensional dS spacetime. We assume that the field is prepared in the Bunch-Davies vacuum state and on the plates a boundary condition is imposed that is a generalization of the perfectly conducting boundary condition for an arbitrary number of spatial dimensions. It is shown that for $D \geq 4$ the background gravitational field essentially changes the behavior of the VEVs at separations between the plates larger than the curvature radius of dS spacetime. At large separations, the Casimir forces are proportional to the inverse fourth power of the distance for all values of spatial dimension $D \geq 3$. For $D \geq 4$ this behavior is in sharp contrast with the case of plates in Minkowski bulk where the force decays as the inverse $(D+1)$ th power of the distance.
\end{abstract}

PACS numbers: 04.62.+v, 04.20.Gz, 04.50.-h, 11.10.Kk

\section{Introduction}

The nontrivial properties of the quantum vacuum are manifested in its response to external influences. In the present paper, for the electromagnetic vacuum, we consider a problem with two kinds of influences: background gravitational field and boundaries constraining the zero-point fluctuations of the electromagnetic field. In order to have an exactly solvable problem we study highly symmetric bulk and boundary geometries. Namely, we consider the de Sitter (dS) spacetime as a background geometry and two parallel plates with perfectly conducting boundary conditions. By taking into account that the higher-dimensional models play an important role in high-energy physics, in particular, in string theories and in supergravity, we shall assume a general value of the spatial dimension $D$. The dS spacetime is among the most popular backgrounds in the gravitational physics. This geometry is of key importance in the inflationary cosmology [1, according to which at the early stages of the cosmological expansion our Universe passed through a phase with the geometry well approximated by dS spacetime. Quantum fluctuations during an inflationary epoch generate inhomogeneities which play a central role in the formation of large scale cosmic structures. From the other side, at the present epoch the Universe is accelerating and the expansion can be

\footnotetext{
*E-mail: anna.kotanjyan@ysu.am

${ }^{\dagger}$ E-mail: saharian@ysu.am
} 
well approximated by a homogeneous and isotropic model with a positive cosmological constant as the dark energy. In this case, the $\mathrm{dS}$ spacetime is a future attractor for the large scale geometry of the Universe. Consequently, the investigation of physical effects in dS background is important for understanding the both early and late stages of the Universe expansion.

In quantum field theory, the interaction of fluctuating quantum fields with a classical background gravitational field leads to the polarization of the vacuum. For the dS geometry this effect has been widely discussed in the literature (for reviews see [2]). The presence of boundaries with prescribed boundary conditions on the field operator gives rise to another type of vacuum polarization known as the Casimir effect [3]. The boundaries can have different physical natures like macroscopic bodies in QED, interfaces separating different phases, horizons in gravitational physics, the branes in higher-dimensional field-theoretical models and in string theories. Historically, the first example of the Casimir effect has been considered for the electromagnetic field in the geometry of two neutral parallel metallic plates [4]. Casimir showed that the plates attract each other through the force which is proportional to the inverse fourth power of the distance between them. The vacuum energy density and the stresses are located in the region between the plates and their distribution in this region is uniform [5]. The latter property is a consequence of the conformal invariance of the electromagnetic field in 4-dimensional spacetime. The vacuum expectation values (VEVs) of the mean electric and magnetic field squared have been discussed in [6, 7]. These VEVs are not uniform and they diverge on the plates. The Casimir densities for the electromagnetic field in an arbitrary number of spatial dimensions $D$ are investigated in [8]. For $D>3$, the electromagnetic field is not conformally invariant and the distributions of the vacuum energy density and of the stresses parallel to the plates are not uniform (see below). In all these considerations of the electromagnetic Casimir effect for parallel conducting plates the background geometry was Minkowskian. The Casimir force with the same geometry of boundaries and in the presence of an extra dimension compactified on a circle is investigated in [9].

An important direction in the investigations of the Casimir effect is the explicit dependence of the characteristics of the vacuum state on the geometry of the background spacetime. The scalar Casimir densities in dS spacetime, induced by planar and spherical boundaries with Robin boundary conditions, are studied in [10] (special cases of conformally and minimally coupled massless scalar fields are considered in [11]). It has been shown that the background gravitational field decisively influences the VEVs of physical observables at distances from the boundaries larger than the curvature scale of the dS spacetime. For the same background, similar features were observed in the topological Casimir effect, induced by toroidal compactification of spatial dimensions [12]. The scalar vacuum densities in background spacetimes with dS and anti-de Sitter (AdS) bubbles are considered in [13. The electromagnetic Casimir densities for a conducting plate in dS spacetime have been investigated in [14]. The case of the electromagnetic field in background of FriedmannRobertson-Walker cosmological models with power-law scale factors is discussed in [15] (for the electromagnetic Casimir effect in Randall-Sundrum braneworld models on AdS bulk see [16] and references therein).

The present paper is organized as follows. In the next section, by using the complete set of the mode functions for the electromagnetic field, we construct the two-point function for the electromagnetic field tensor in the geometry of two conducting plates in background of $(D+1)$-dimensional $\mathrm{dS}$ spacetime. This function is presented as the sum of boundary-free two-point functions corresponding to an infinite sequence of image sources. The mean electric field squared and the VEV of the energy-momentum tensor in the region between two plates are investigated in Section 3 . Various limiting cases are discussed in detail. The Casimir forces acting on the plates are studied in Section 4, The main conclusions of the paper are summarized in Section 5 . 


\section{Electromagnetic two-point functions}

Consider two perfectly conducting plates in the background of $(D+1)$-dimensional dS spacetime. In inflationary coordinates, the geometry is described by the line element

$$
d s^{2}=d t^{2}-e^{2 t / \alpha}(d \mathbf{z})^{2}, \mathbf{z}=\left(z^{1}, z^{2}, \ldots, z^{D}\right),
$$

where the parameter $\alpha$ is expressed in terms of the positive cosmological constant $\Lambda$ by the relation $\alpha^{2}=D(D-1) /(2 \Lambda)$. Introducing the conformal time $\tau$ in accordance with $\tau=-\alpha e^{-t / \alpha},-\infty<$ $\tau<0$, the metric tensor is written in the conformally flat form: $g_{\mu \nu}=(\alpha / \tau)^{2} \eta_{\mu \nu}$ with $\eta_{\mu \nu}$ being the metric tensor for Minkowski spacetime. In what follows we shall work in spacetime coordinates $(\tau, \mathbf{z})$.

We assume that the plates are placed at $z^{D}=0$ and $z^{D}=L$ and the electromagnetic field is prepared in the Bunch-Davies vacuum state $|0\rangle$. Among the set of dS-invariant quantum states, the Bunch-Davies vacuum is the only one for which the ultraviolet behavior of the two-point functions is the same as in Minkowski spacetime. On the plates the field obeys the boundary condition [17] $n^{\nu_{1}{ }^{*}} F_{\nu_{1} \cdots \nu_{D-1}}=0$, where ${ }^{*} F_{\nu_{1} \cdots \nu_{D-1}}$ is dual to the electromagnetic field tensor $F_{\mu \nu}=\partial_{\mu} A_{\nu}-\partial_{\nu} A_{\mu}$ and $n^{\mu}$ is the normal to the plates. This condition is a generalization of the perfectly conducting boundary condition in electrodynamics for models with an arbitrary number of spatial dimensions. For the coordinates and for the momentum components parallel to the plates we shall use the notations $\mathbf{z}_{\|}=\left(z^{1}, \ldots, z^{D-1}\right)$ and $\mathbf{k}_{\|}=\left(k_{1}, \ldots, k_{D-1}\right)$. In the regions $z^{D}<0$ and $z^{D}>L$ the VEVs are the same as those in the geometry of a single plate located at $z^{D}=0$ and $z^{D}=L$, respectively. This geometry is considered in [14] and below we shall be mainly concerned with the region between the plates, $0<z^{D}<L$. In this region, the mode functions for the vector potential $A_{\mu}(x), x=(\tau, \mathbf{z})$, realizing the Bunch-Davies vacuum state and obeying the gauge conditions $A_{0}=0, \nabla_{\mu} A^{\mu}=0$, have the form

$$
A_{(\sigma \mathbf{k}) l}(x)=i C \epsilon_{(\sigma) l} \eta^{D / 2-1} H_{D / 2-1}^{(1)}(k \eta) \sin \left(k_{D} z^{D}\right) e^{i \mathbf{k}_{\|} \cdot \mathbf{z}_{\|}},
$$

for the components with $l=1, \ldots, D-1$, and

$$
A_{(\sigma \mathbf{k}) D}(x)=C \epsilon_{(\sigma) D} \eta^{D / 2-1} H_{D / 2-1}^{(1)}(k \eta) \cos \left(k_{D} z^{D}\right) e^{i \mathbf{k}_{\|} \cdot \mathbf{z}_{\|}},
$$

where $\eta=|\tau|, \mathbf{k}=\left(\mathbf{k}_{\|}, k_{D}\right), k=\sqrt{k_{D}^{2}+\mathbf{k}_{\|}^{2}}$ and $\mathbf{k}_{\|} \cdot \mathbf{z}_{\|}=\sum_{l=1}^{D-1} k_{l} z^{l}$. In these expressions, $H_{D / 2-1}^{(1)}(k \eta)$ is the Hankel function of the first kind and for the polarization vectors $\epsilon_{(\sigma) l}$, with $\sigma=1, \ldots, D-1$, one has the transverse condition $\sum_{l=1}^{D} \epsilon_{(\sigma) l} k_{l}=0$ and the relations

$$
\sum_{l=1}^{D} \epsilon_{(\sigma) l} \epsilon_{\left(\sigma^{\prime}\right) l}=\delta_{\sigma \sigma^{\prime}}, \sum_{\sigma=1}^{D-1} \epsilon_{(\sigma) l} \epsilon_{(\sigma) m}=\delta_{l m}-\frac{k_{l} k_{m}}{k^{2}} .
$$

The modes (2) and (3) obey the boundary condition on the plate $z^{D}=0$. From the boundary condition at $z^{D}=L$ for the eigenvalues of the component of the momentum normal to the plates we get

$$
k_{D}=\pi n / L, n=0,1, \ldots
$$

For the normalization coefficient one finds

$$
|C|^{2}=\frac{(2 \pi \alpha)^{3-D}}{2\left(1+\delta_{n 0}\right) L} .
$$

Note that for a general dS-invariant vacuum state, in the mode functions (2) and (3) a linear combination of the Hankel functions $H_{D / 2-1}^{(1)}(k \eta)$ and $H_{D / 2-1}^{(2)}(k \eta)$ appears instead of the function $H_{D / 2-1}^{(1)}(k \eta)$. The choice of the coefficient in the linear combination fixes the vacuum state. 
Having a complete set of mode functions, we can evaluate the two-point function $W_{l m}\left(x, x^{\prime}\right)=$ $\left\langle 0\left|A_{l}(x) A_{m}\left(x^{\prime}\right)\right| 0\right\rangle$ for the vector potential by using the mode-sum formula

$$
W_{l m}\left(x, x^{\prime}\right)=\sum_{\sigma=1}^{D-1} \sum_{n=0}^{\infty} \int d \mathbf{k}_{\|} A_{(\sigma \mathbf{k}) l}(x) A_{(\sigma \mathbf{k}) m}\left(x^{\prime}\right) .
$$

By applying to the sum over $n$ the Poisson resummation formula, this function is presented in the form

$$
W_{l m}\left(x, x^{\prime}\right)=\sum_{n=-\infty}^{\infty} \sum_{j=-1}^{+1} j^{1-\delta_{m D}} W_{l m}^{(0)}\left(x, x_{j, n}^{\prime}\right)
$$

where $W_{l m}^{(0)}\left(x, x^{\prime}\right)$ is the corresponding function in boundary-free dS spacetime and

$$
x_{ \pm 1, n}^{\prime}=\left(\tau^{\prime}, \mathbf{z}_{\|}^{\prime}, \pm z^{D \prime}+2 n L\right) .
$$

In (8), the term $j=+1, n=0$ corresponds to the boundary-free function. The terms $j=-1, n=0$ and $j=-1, n=+1$ correspond to the parts in the two-point function induced by single plates at $z^{D}=0$ and $z^{D}=L$, respectively, when the second plate is absent. The two-point functions for both massive and massless vector fields in dS spacetime are considered in [19]. Recently, it has been shown that the infrared pathologies of the photon two-point function in dS spacetime are purely gauge artifacts [20].

A formula similar to (8) is obtained for the two-point function $W_{l m, p q}\left(x, x^{\prime}\right)=\left\langle 0\left|F_{l m}(x) F_{p q}\left(x^{\prime}\right)\right| 0\right\rangle$ corresponding to the field tensor:

$$
W_{l m, p q}\left(x, x^{\prime}\right)=\sum_{n=-\infty}^{\infty} \sum_{j=-1}^{+1} j^{1-\delta_{p D}} W_{l m, p q}^{(0)}\left(x, x_{j, n}^{\prime}\right),
$$

with $W_{l m, p q}^{(0)}\left(x, x^{\prime}\right)$ being the function for the boundary-free dS geometry. Introducing the notations

$$
B_{D}=(4 \pi)^{(D-1) / 2} \Gamma\left(\frac{D+3}{2}\right), z=1+\frac{\left(\eta-\eta^{\prime}\right)^{2}-|\Delta \mathbf{z}|^{2}}{4 \eta \eta^{\prime}},
$$

with $\Delta \mathbf{z}=\mathbf{z}-\mathbf{z}^{\prime}$, for the components of the boundary-free two-point function one has [14]

$$
\begin{aligned}
W_{0 l, 0 m}^{(0)}\left(x, x^{\prime}\right) & =\frac{\left(\eta \eta^{\prime}\right)^{-2}}{2 B_{D} \alpha^{D-3}}\left[\left(\delta_{l p} \delta_{m q}-\delta_{l m} \delta_{p q}\right) \frac{\Delta z^{p} \Delta z^{q}}{2 \eta \eta^{\prime}} \partial_{z}+(D-1) \delta_{l m}\right] G_{D}(z), \\
W_{l m, 0 p}^{(0)}\left(x, x^{\prime}\right) & =\frac{\left(\eta \eta^{\prime}\right)^{-2}}{B_{D} \alpha^{D-3}} \delta_{[l p} \delta_{m] q} \frac{\Delta z^{q}}{\eta^{\prime}}\left[2+\left(z-\frac{\eta+\eta^{\prime}}{2 \eta}\right) \partial_{z}\right] F_{D}(z), \\
W_{l m, p q}^{(0)}\left(x, x^{\prime}\right) & =\frac{\left(\eta \eta^{\prime}\right)^{-2}}{B_{D} \alpha^{D-3}}\left(\delta_{[l r} \delta_{m][p} \delta_{q] s} \frac{\Delta z^{r} \Delta z^{s}}{\eta \eta^{\prime}} \partial_{z}+2 \delta_{[l p} \delta_{m] q}\right) F_{D}(z),
\end{aligned}
$$

where $l, m, p, q=1,2, \ldots, D$, and the expression for the component $W_{0 p, l m}^{(0)}\left(x, x^{\prime}\right)$ is obtained from that for $W_{l m, 0 p}^{(0)}\left(x, x^{\prime}\right)$ by changing the sign and by the interchange $\eta \rightleftarrows \eta^{\prime}$. In (12), the square brackets enclosing the indices mean the antisymmetrization over these indices and we have defined the functions

$$
\begin{aligned}
F_{D}(z) & =\Gamma(D) F\left(D, 2 ; \frac{D+3}{2} ; z\right), \\
G_{D}(z) & =2 \Gamma(D-1) F\left(D-1,3 ; \frac{D+3}{2} ; z\right),
\end{aligned}
$$


with $F(a, b, c ; z)$ being the hypergeometric function.

In what follows, we shall need the asymptotic expressions of the functions (13) (see also [14]). For $0<1-z \ll 1$, by using the asymptotic expansion for the hypergeometric function [21], to the leading order one has

$$
F_{D}(z) \approx G_{D}(z) \approx(D+1) \frac{\Gamma^{2}((D+1) / 2)}{2(1-z)^{(D+1) / 2}} .
$$

In the region $z \ll-1$, by making use of the linear transformation formulas relating the hypergeometric functions with the arguments $z$ and $1 / z$ [21], for the function $F_{D}(z)$ we get

$$
\begin{aligned}
& F_{D}(z) \approx\left(D^{2}-1\right) \frac{\Gamma(D-2)}{4(-z)^{2}}, D>2, \\
& F_{D}(z) \approx \frac{\Gamma((D+3) / 2)}{2^{D-1} \sqrt{\pi}(-z)^{D}} \Gamma(D) \Gamma(1-D / 2), D<2 .
\end{aligned}
$$

For $D=2$ one has $F_{D}(z) \approx 3(-z)^{-2}[\ln (-4 z)-1] / 4$. In a similar way, for the function $G_{D}(z)$ one finds

$$
\begin{aligned}
& G_{D}(z) \approx \frac{\left(D^{2}-1\right)(D-3)}{4(-z)^{3}} \Gamma(D-4), D>4, \\
& G_{D}(z) \approx \Gamma(D-1) \frac{\Gamma((D+3) / 2) \Gamma(2-D / 2)}{2^{D-3} \sqrt{\pi}(-z)^{D-1}}, D<4,
\end{aligned}
$$

and $G_{4}(z) \approx 3(-z)^{-3}[2 \ln (-4 z)-3]$ for $D=4$.

On the base of the representation (10), the two-point function can be presented in the decomposed form

$$
W_{l m, p q}\left(x, x^{\prime}\right)=W_{l m, p q}^{(0)}\left(x, x^{\prime}\right)+W_{l m, p q}^{(b)}\left(x, x^{\prime}\right),
$$

where the boundary-induced part $W_{l m, p q}^{(b)}\left(x, x^{\prime}\right)$ is given by the right-hand side of (10) omitting the term with $j=+1, n=0$. Here we consider a free field theory and all the properties of the vacuum state are encoded in the two-point functions. In the next sections we consider the Casimir densities and the forces acting on the plates.

\section{Casimir densities}

\subsection{Mean field squared}

We start the investigation of the VEVs in the region between the plates by evaluating the mean electric field squared. For $D=3$ it determines the Casimir-Polder force acting on a polarizable particle placed near the plates. The VEV of the electric field squared is obtained from the two-point function in the coincidence limit of the arguments:

$$
\left\langle E^{2}\right\rangle=-g^{00} g^{l m} \lim _{x^{\prime} \rightarrow x} W_{0 l, 0 m}\left(x, x^{\prime}\right) .
$$

For points away from the plates, the divergences in this limit are contained in the boundary-free part and the renormalization is needed for this part only. The renormalized VEV is presented in the form

$$
\left\langle E^{2}\right\rangle=\left\langle E^{2}\right\rangle_{0}+\left\langle E^{2}\right\rangle_{\mathrm{b}}
$$

where $\left\langle E^{2}\right\rangle_{0}$ is the renormalized VEV in boundary-free dS spacetime and the part $\left\langle E^{2}\right\rangle_{\mathrm{b}}$ is induced by the plates. The latter is obtained by the formula similar to (18) with the function $W_{0 l, 0 m}^{(b)}\left(x, x^{\prime}\right)$. 
Introducing the notations

$$
\begin{aligned}
& u_{n}=1-(n L / \eta)^{2}, \\
& v_{n}=1-\left(z^{D}-n L\right)^{2} / \eta^{2},
\end{aligned}
$$

in the region between the plates the boundary-induced contribution is presented in the form

$$
\begin{aligned}
\left\langle E^{2}\right\rangle_{\mathrm{b}}= & \frac{D-1}{2 B_{D^{\alpha}}{ }^{D+1}}\left\{2 \sum_{n=1}^{+\infty}\left[2\left(u_{n}-1\right) \partial_{u_{n}}+D\right] G_{D}\left(u_{n}\right)\right. \\
& \left.-\sum_{n=-\infty}^{+\infty}\left[2\left(v_{n}-1\right) \partial_{v_{n}}+D-2\right] G_{D}\left(v_{n}\right)\right\} .
\end{aligned}
$$

This contribution depends on $z^{D}, L$ and $\eta$ through the combinations $z^{D} / \eta$ and $L / \eta$. These ratios are the proper distance from the left plate and the proper separation between the plates measured in units of the $\mathrm{dS}$ curvature scale $\alpha$. This property is a consequence of the maximal symmetry of the background spacetime and of the Bunch-Davies vacuum state. In (21), the $n=0$ and $n=1$ terms of the last series are the contributions induced by single plates at $z^{D}=0$ and $z^{D}=L$, respectively, when the second plate is absent. The surface divergences are contained in these two terms and, hence, for points near the plates they dominate in the VEV of the electric field squared. In particular, near the left plate, $z^{D} / \eta \ll 1$, by using (14), to the leading order one has

$$
\left\langle E^{2}\right\rangle_{\mathrm{b}} \approx \frac{3(D-1) \Gamma((D+1) / 2)}{2(4 \pi)^{(D-1) / 2}\left(\alpha z^{D} / \eta\right)^{D+1}} .
$$

As is seen, near the plates the mean field squared is positive.

For $D=3$, by using $G_{3}(z)=2 /(1-z)^{2}$, from the general formula one finds

$$
\left\langle E^{2}\right\rangle_{\mathrm{b}}=\frac{3 \zeta_{4}\left(z^{D} / L\right)-2 \zeta(4)}{4 \pi(\alpha L / \eta)^{4}}
$$

with $\zeta(s)$ being the Riemann zeta function, $\zeta(4)=\pi^{4} / 90$, and we have introduced the function

$$
\zeta_{p}(x)=\sum_{n=-\infty}^{\infty} \frac{1}{|n-x|^{p}}=\zeta(p, x)+\zeta(p, 1-x) .
$$

Here $\zeta(p, x)$ is the Hurwitz zeta function. The electromagnetic field is conformally invariant for $D=3$ and the expression (23) is related to the corresponding result for the plates in Minkowski spacetime with the separation $L$ by the formula $\left\langle E^{2}\right\rangle_{\mathrm{b}}=(\eta / \alpha)^{4}\left\langle E^{2}\right\rangle_{\mathrm{b}}^{(M)}$. In the case $D=5$, by making use of the expression $G_{5}(z)=12 /(1-z)^{3}$, we get

$$
\left\langle E^{2}\right\rangle_{\mathrm{b}}=\frac{3 \zeta_{6}\left(z^{D} / L\right)-2 \zeta(6)}{4 \pi^{2}(\alpha L / \eta)^{6}}
$$

with $\zeta(6)=\pi^{6} / 945$.

Let us consider the mean field squared in the limits of small and large proper separations between the plates. When the proper separation of the plates is small compared to the dS curvature radius, $L / \eta \ll 1$, the curvature effects are small, and one can see that, to the leading order, the VEV of the electric field squared coincides with that for plates in Minkowski spacetime with the separation $\alpha L / \eta$ :

$$
\left\langle E^{2}\right\rangle_{\mathrm{b}} \approx \frac{(D-1) \Gamma((D+1) / 2)}{2(4 \pi)^{(D-1) / 2}(\alpha L / \eta)^{D+1}}\left[3 \zeta_{D+1}\left(z^{D} / L\right)-2 \zeta(D+1)\right] .
$$


It can be seen that the expression in the right-hand side of (26) is obtained from the results of 8 ] for $\left\langle E_{D}^{2}\right\rangle$ and $\left\langle E_{l}^{2}\right\rangle, l=1, \ldots, D-1$, in Minkowski bulk. For $D=3$ and $D=5$, the leading term (26) , coincides with the exact expressions (23) and (25). By taking into account that $\zeta_{p}(x)>\zeta(p)$ for $0<x<1$, from (26) we conclude that at small separations the mean electric field squared is positive.

When the proper separation between the plates is large, $L / \eta \gg 1$, the effects from the curvature of the background spacetime are essential. Assuming that $z^{D}, L-z^{D} \gg \eta$ and by using the asymptotic formulas (16), we get

$$
\left\langle E^{2}\right\rangle_{\mathrm{b}} \approx \frac{(D-1) \Gamma(D / 2-2)}{8 \pi^{D / 2} \alpha^{D+1}(L / \eta)^{6}}\left[(D-6) \zeta(6)-(D / 2-4) \zeta_{6}\left(z^{D} / L\right)\right]
$$

for $D>4$ and

$$
\left\langle E^{2}\right\rangle_{\mathrm{b}} \approx-\frac{2^{3-2 D} \Gamma(D) \Gamma(2-D / 2)}{\pi^{D / 2} \alpha^{D+1}(L / \eta)^{2(D-1)}}\left[2(D-2) \zeta(2(D-1))-D \zeta_{2(D-1)}\left(z^{D} / L\right)\right],
$$

for $D<4$. In the case $D=4$ the asymptotic has the form

$$
\left\langle E^{2}\right\rangle_{\mathrm{b}} \approx-\frac{3 \alpha^{-5}}{\pi^{2}(L / \eta)^{6}}\left[\sum_{n=1}^{+\infty} \frac{\ln (n L / \eta)}{n^{6}}-\sum_{n=-\infty}^{+\infty} \frac{\ln \left(\left|z^{D}-n L\right| / \eta\right)}{\left(z^{D} / L-n\right)^{6}}\right] .
$$

Again, for $D=3$ and $D=5$, the expressions (28) and (27) coincide with the exact results (23) and (25), respectively. With dependence of $D$ and $z^{D} / L$, the boundary-induced $\operatorname{VEV}\left\langle E^{2}\right\rangle_{\mathrm{b}}$ can be either positive or negative.

\section{$3.2 \quad$ Energy-momentum tensor}

Another important characteristic of the vacuum state is the VEV of the energy-momentum tensor. It describes the local structure of the vacuum state and plays an important role in modeling the self-consistent dynamic involving the gravitational field.

Having the two-point function for the electromagnetic field tensor we can evaluate the VEV of the energy-momentum tensor by making use of the formula

$$
\left\langle T_{\mu \nu}\right\rangle=\frac{1}{4 \pi} g^{\beta \rho} \lim _{x^{\prime} \rightarrow x}\left[-W_{\mu \beta, \nu \rho}\left(x, x^{\prime}\right)+\frac{1}{4} g_{\mu \nu} g^{\sigma s} W_{\sigma \beta, s \rho}\left(x, x^{\prime}\right)\right] .
$$

Of course, the expression in the right-hand side is divergent and some renormalization procedure is needed. The important thing here is that we have already decomposed the two-point functions. This allows us to have a similar decomposition of the vacuum energy-momentum tensor:

$$
\left\langle T_{\mu}^{\nu}\right\rangle=\left\langle T_{\mu}^{\nu}\right\rangle_{0}+\left\langle T_{\mu}^{\nu}\right\rangle_{\mathrm{b}}
$$

with the boundary-free, $\left\langle T_{\mu}^{\nu}\right\rangle_{0}$, and boundary-induced, $\left\langle T_{\mu}^{\nu}\right\rangle_{\mathrm{b}}$, parts. For points outside the plates the local geometry is not changed by the presence of the plates and the divergences in $\left\langle T_{\mu}^{\nu}\right\rangle$ and $\left\langle T_{\mu}^{\nu}\right\rangle_{0}$ are the same. Hence, with the decomposition (31), for the points outside the plate the renormalization of $\left\langle T_{\mu}^{\nu}\right\rangle$ is reduced to the one for boundary-free dS spacetime. Because of the maximal symmetry of de Sitter spacetime and the Bunch-Davies vacuum state, the corresponding $\mathrm{VEV}$ of the energy-momentum tensor is proportional to the metric tensor: $\left\langle T_{\mu}^{\nu}\right\rangle_{0}=\operatorname{const} \cdot \delta_{\mu}^{\nu}$.

Our main interest here is the boundary-induced part in the VEV of the energy-momentum tensor. It is obtained by using the formula (30) with the function $W_{\mu \beta, \nu \rho}^{(b)}\left(x, x^{\prime}\right)$. In the region 
between the plates, for the diagonal components of vacuum energy-momentum tensor one finds (no summation over $\mu$ )

$$
\left\langle T_{\mu}^{\mu}\right\rangle_{\mathrm{b}}=\frac{D-1}{16 \pi \alpha^{D+1} B_{D}}\left[2 \sum_{n=1}^{+\infty} U^{(\mu)}\left(u_{n}\right)+\sum_{n=-\infty}^{+\infty} V^{(\mu)}\left(v_{n}\right)\right] .
$$

Here we have introduced new functions

$$
\begin{aligned}
U^{(0)}(u) & =\left[2(u-1) \partial_{u}+D\right] F_{D}^{(+)}(u), \\
U^{(l)}(u) & =\left[2 \frac{D-3}{D-1}(u-1) \partial_{u}+D-2\right] F_{D}^{(-)}(u)-2 F_{D}(u), \\
U^{(D)}(u) & =-\left[2(u-1) \partial_{u}+D-2\right] F_{D}^{(+)}(u)+2(D-3) F_{D}(u),
\end{aligned}
$$

and

$$
\begin{aligned}
V^{(0)}(u) & =\left[2(u-1) \partial_{u}+D-2\right] F_{D}^{(-)}(u)-2(D-3) F_{D}(u), \\
V^{(l)}(u) & =2 \frac{D-3}{D-1}\left[(u-1) \partial_{u} F_{D}^{(+)}(y)+2 F_{D}(u)\right]-(D-4) F_{D}^{(-)}(u), \\
V^{(D)}(u) & =-\left[2(u-1) \partial_{u}+D\right] F_{D}^{(-)}(u),
\end{aligned}
$$

with $l=1,2, \ldots, D-1$ and

$$
F_{D}^{( \pm)}(u)=F_{D}(u) \pm G_{D}(u)
$$

The expression (32) is symmetric with respect to the plane $z^{D}=L / 2$. In (32), the contributions of the terms $V^{(\mu)}\left(v_{0}\right)$ and $V^{(\mu)}\left(v_{+1}\right)$ correspond to the geometries with single plates at $z^{D}=0$ and $z^{D}=L$, respectively, when the second plate is absent. For $D \geqslant 3$, the divergences on the plates come from these terms.

The problem under consideration is not symmetric under the translations along the time coordinate and along the $z^{D}$ coordinate. As a result of this, the vacuum energy-momentum tensor has nonzero off-diagonal component

$$
\left\langle T_{0}^{D}\right\rangle_{\mathrm{b}}=-\frac{(D-1) L / \eta}{4 \pi B_{D} \alpha^{D+1}} \sum_{n=-\infty}^{+\infty}\left(z^{D} / L-n\right)\left[\left(v_{n}-1\right) \partial_{v_{n}}+2\right] F_{D}\left(v_{n}\right),
$$

which describes the energy flux along the direction normal to the plates. The off-diagonal component is antisymmetric with respect to the plane $z^{D}=L / 2$ and, hence, it vanishes at $z^{D}=L / 2$. The $n=0$ term in the right-hand side of (36) gives the energy flux induced by the plate at $z^{D}=0$ when the right plate is absent. Similarly, the $n=1$ term presents the energy flux in the geometry of a single plate $z^{D}=L$. The boundary-induced VEVs (32) and (36) depend on $L, z^{D}$, and $\eta$ through the combinations $L / \eta$ and $z^{D} / \eta$. As we have noticed before, they are the proper separation between the plates and the proper distance from the left plate measured in units of $\alpha$.

For $D \geqslant 4$, for points near the plates the VEVs are dominated by single plate parts. In particular, near the left plate, to the leading order one has [14] (no summation over $l=0, \ldots, D-1$ )

$$
\left\langle T_{l}^{l}\right\rangle_{\mathrm{b}} \approx-\frac{\eta}{z^{D}}\left\langle T_{0}^{D}\right\rangle_{\mathrm{b}} \approx \frac{D-1}{\left(z^{D} / \eta\right)^{2}}\left\langle T_{D}^{D}\right\rangle_{\mathrm{b}} \approx-\frac{(D-3)(D-1) \Gamma((D+1) / 2)}{2(4 \pi)^{(D+1) / 2} \alpha^{D+1}\left(z^{D} / \eta\right)^{D+1}} .
$$

The asymptotic expressions near the right plate are obtained by the replacement $z^{D} \rightarrow L-z^{D}$. As is seen, near the plates the diagonal components of the boundary-induced vacuum energy-momentum tensor are negative and the energy flux is directed from the plates. 
By using $F_{3}(z)=2 /(1-z)^{2}$ and the expression for $G_{3}(y)$ given above, we can see that for $D=3$ one has

$$
\left\langle T_{\mu}^{\nu}\right\rangle_{\mathrm{b}}=-\frac{\pi^{2}}{720(\alpha L / \eta)^{4}} \operatorname{diag}(1,1,1,-3)
$$

This expression coincides with the standard result for the plates in Minkowski bulk [5] with the separation $\alpha L / \eta$. Again, this property is a consequence of the conformal invariance of the electromagnetic field in $D=3$. Relatively simple expressions are also obtained for $D=5$. In this case for the diagonal components we get (no summation over $\mu$ )

$$
\left\langle T_{\mu}^{\mu}\right\rangle_{\mathrm{b}}=\frac{(\alpha L / \eta)^{-6}}{32 \pi^{3}} \sum_{m=2,3} \frac{(-1)^{m}}{(L / \eta)^{2(m-3)}}\left[2 U^{(\mu, m)} \zeta(2 m)+V^{(\mu, m)} \zeta_{2 m}\left(z^{D} / L\right)\right],
$$

with the coefficients

$$
\begin{aligned}
& \left(U^{(0,2)}, U^{(1,2)}, U^{(5,2)}\right)=(1,-1,5),\left(U^{(0,3)}, U^{(1,3)}, U^{(5,3)}\right)=(2,2,-10), \\
& \left(V^{(0,2)}, V^{(1,2)}, V^{(5,2)}\right)=(-5,-1,-1),\left(V^{(0,3)}, V^{(1,3)}, V^{(5,3)}\right)=(4,4,0) .
\end{aligned}
$$

For $D=5$ the expression of the off-diagonal component takes the form

$$
\left\langle T_{0}^{D}\right\rangle_{\mathrm{b}}=\frac{\zeta\left(5, z^{D} / L\right)-\zeta\left(5,1-z^{D} / L\right)}{8 \pi^{3} \alpha(\alpha L / \eta)^{5}} .
$$

Now we turn to the investigation of the asymptotic behavior for the components of VEV of the energy-momentum tensor. First, we consider the limit when the proper separation between the plates is small compared with the $\mathrm{dS}$ curvature radius, $L / \eta \ll 1$. In this limit the gravitational effects in the boundary-induced parts of the diagonal components are subdominant and to the leading order one finds (no summation over $\mu$ )

$$
\left\langle T_{\mu}^{\mu}\right\rangle_{\mathrm{b}} \approx-\frac{(D-1) \Gamma((D+1) / 2)}{2(4 \pi)^{(D+1) / 2}(\alpha L / \eta)^{D+1}}\left[2 \zeta(D+1)+(D-3) \zeta_{D+1}\left(z^{D} / L\right)\right],
$$

for $\mu=0,1, \ldots, D-1$, and

$$
\left\langle T_{D}^{D}\right\rangle_{\mathrm{b}} \approx \frac{D(D-1) \Gamma((D+1) / 2)}{(4 \pi)^{(D+1) / 2}(\alpha L / \eta)^{D+1}} \zeta(D+1) .
$$

The expressions (42) and (43) coincide with the corresponding results for parallel plates in Minkowski bulk with the separation $\alpha L / \eta$. For the energy flux, to the leading order, one gets

$$
\left\langle T_{0}^{D}\right\rangle \approx \frac{(D-1)(D-3) \Gamma((D+1) / 2)}{2(4 \pi)^{(D+1) / 2} \alpha(\alpha L / \eta)^{D}}\left[\zeta\left(D, z^{D} / L\right)-\zeta\left(D, 1-z^{D} / L\right)\right] .
$$

The flux is positive for $0<z^{D}<L / 2$ and negative for $L / 2<z^{D}<L$. Note that for $D=5$ the expression in the right-hand side of (44) coincides with the exact result (41).

In the opposite limit, when the separation between the plates is large compared to the dS curvature radius, that corresponds to $L / \eta \gg 1$, and assuming also $z^{D}, L-z^{D} \gg \eta$, for $D \geqslant 4$ to the leading order one finds (no summation over $\mu$ )

$$
\left\langle T_{\mu}^{\mu}\right\rangle_{\mathrm{b}} \approx \frac{(D-1) \Gamma(D / 2-1)}{2^{6} \pi^{D / 2+1} \alpha^{D+1}(L / \eta)^{4}}\left[2 U_{\mu} \zeta(4)+V_{\mu} \zeta_{4}\left(z^{D} / L\right)\right],
$$

with the notations

$$
\begin{aligned}
& U_{0}=D-4, U_{l}=D-4-4 \frac{D-3}{D-1}, U_{D}=D, \\
& V_{0}=-D, V_{l}=V_{D}=4-D,
\end{aligned}
$$


and $l=1, \ldots, D-1$. For the off-diagonal component, in the same limit, we get

$$
\left\langle T_{0}^{D}\right\rangle_{\mathrm{b}} \approx \frac{(D-1) \Gamma(D / 2-1)}{2^{4} \pi^{D / 2+1} \alpha^{D+1}(L / \eta)^{5}}\left[\zeta\left(5, z^{D} / L\right)-\zeta\left(5,1-z^{D} / L\right)\right]
$$

Again, the flux is positive for $0<z^{D}<L / 2$ and negative for $L / 2<z^{D}<L$.

In figure 1 we have plotted the diagonal (left panel) and the off-diagonal (right panel) components of the vacuum energy-momentum tensor as functions of $z^{D} / L$ for $D=4 \mathrm{dS}$ spacetime. For the left panel we have taken the separation between the plates corresponding to $L / \eta=2$ and the lower, middle, upper curves correspond to the components $\left\langle T_{0}^{0}\right\rangle_{\mathrm{b}},\left\langle T_{1}^{1}\right\rangle_{\mathrm{b}},\left\langle T_{4}^{4}\right\rangle_{\mathrm{b}}$, respectively. On the left panel the energy flux is plotted for $L / \eta=0.75,1,1.5$ (the curves from the right to the left in the regions $0<z^{D} / L<1 / 2, z^{D} / L>1$, and the curves from the left to the right in the regions $\left.z^{D} / L<0,1 / 2<z^{D} / L<1\right)$.
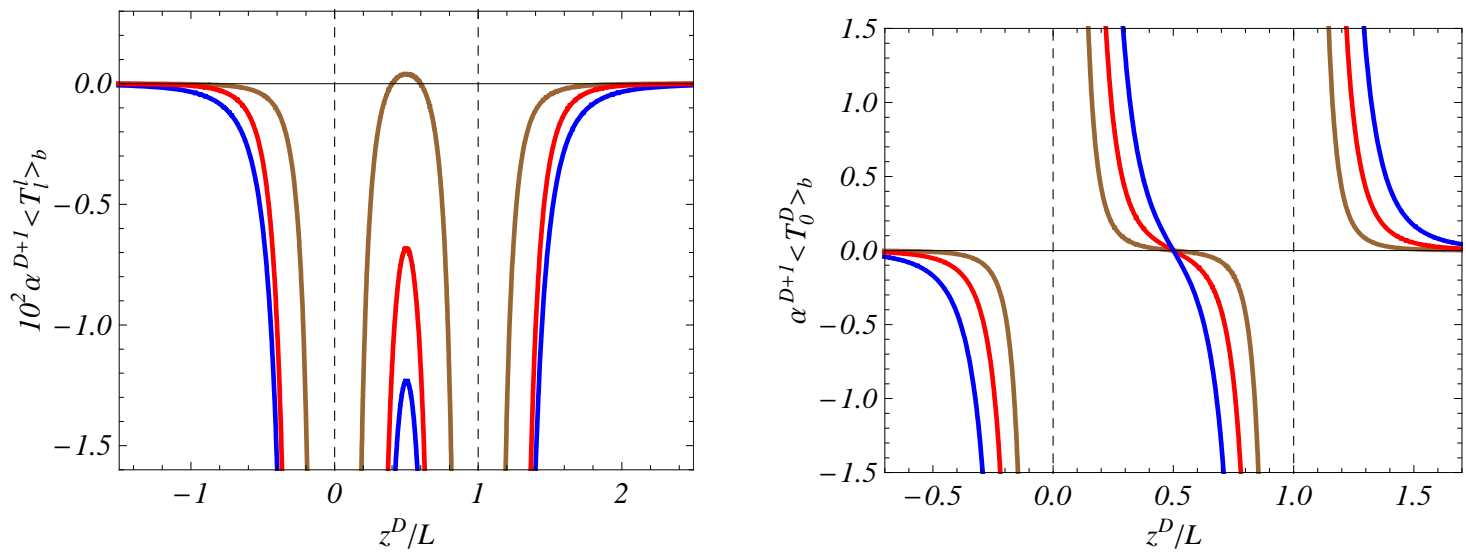

Figure 1: The VEVs of the diagonal (left panel) and off-diagonal components of the energymomentum tensor as functions of the rescaled coordinate $z^{D} / L$ in the geometry of two conducting plates for $D=4$. The vertical dashed lines correspond to the locations of the plates.

\section{Casimir forces}

From the symmetry of the problem under consideration it follows that the Casimir forces acting on the left and right plates are equal in magnitude. We shall consider the force acting per unit surface of the left plate (the vacuum effective pressure on the plate). Having the normal stress, the effective pressure $P$ is evaluated as $P=-\left\langle T_{D}^{D}\right\rangle_{z^{D}=0}$. In the discussion above we have decomposed the normal stress into the single plate and second plate-induced contributions. Consequently, the Casimir force is decomposed into the self-action and interaction parts. The self-action force on the right-hand side of the plate at $z^{D}=0$ is determined by the $n=0$ term in the expression (32) for the component with $\mu=D$. Because of the surface divergences in the boundary-induced VEVs this force is divergent. However, by the symmetry, the self-forces acting on a single plate from the left- and right-hand sides cancel each other and the net self-action force vanishes (note that this would not be the case for curved boundaries).

The interaction part of the vacuum pressure, denoted here as $P^{(\mathrm{int})}$, is induced by the presence of the second plate. It is directly obtained from the expression (32) for $\left\langle T_{D}^{D}\right\rangle$ omitting the term $n=0$ :

$$
P^{(\mathrm{int})}=\frac{(D-1) \alpha^{-D-1}}{(4 \pi)^{(D+1) / 2} \Gamma((D+3) / 2)} \sum_{n=1}^{\infty}\left\{2\left[\left(u_{n}-1\right) \partial_{u_{n}}+1\right] F_{D}\left(u_{n}\right)-G_{D}\left(u_{n}\right)\right\}
$$


where $u_{n}$ is defined by (20). Relatively simple expressions are obtained for odd values of the spatial dimension. In particular, in the cases $D=3$ and $D=5$ one finds

$$
\begin{aligned}
& P^{(\mathrm{int})}=-\frac{\pi^{2}}{240(\alpha L / \eta)^{4}}, D=3 \\
& P^{(\mathrm{int})}=-\frac{\pi \alpha^{-6}}{360(L / \eta)^{4}}\left[1+\frac{5 \pi^{2}}{21(L / \eta)^{2}}\right], D=5 .
\end{aligned}
$$

For small separations between the plates, that correspond to $L / \eta \ll 1$, from (48) one has the asymptotic expression

$$
P^{(\mathrm{int})} \approx-\frac{D(D-1) \Gamma((D+1) / 2)}{(4 \pi)^{(D+1) / 2}(\alpha L / \eta)^{D+1}} \zeta(D+1) .
$$

This leading term coincides with the expression of the force in Minkowski bulk with the separation between the plates equal to $\alpha L / \eta$ (proper distance between the plates in dS spacetime). At large separations between plates one has $L / \eta \gg 1$ and for $D \geqslant 4$ to the leading order we get

$$
P^{(\mathrm{int})} \approx-\frac{(D-1) \Gamma(D / 2-1)}{720 \pi^{D / 2-3} \alpha^{D+1}(L / \eta)^{4}} .
$$

In both cases of (50) and (51) we have $P^{(\mathrm{int})}<0$ and the corresponding forces are attractive. It is of interest to note that at large separations the force decays as the inverse forth power of the proper separation for all values of $D \geqslant 3$. As it has been shown in [10], for a massive scalar field on dS background, depending on the values of the curvature coupling parameter and the mass of the field, two different regimes are realized, which exhibit monotonic and oscillatory decay of the Casimir forces at large separations. In the physical problem under consideration the quantum field is massless and the forces decay monotonically.

By taking into account that $\eta=\alpha e^{-t / \alpha}$, we see that the formulae (50) and (51) describe the behavior of the Casimir forces at early and late stages of the cosmological expansion for a fixed value of the ratio $L / \alpha$. At early stages of the expansion, $t \rightarrow-\infty$, the Casimir force behaves as $P^{(\text {int })} \propto e^{-(D+1) t / \alpha}$. At late stages, $t \rightarrow+\infty$, the force decays as $P^{(\text {int })} \propto e^{-4 t / \alpha}$ for all values of the spatial dimension $D \geqslant 3$.

For the study of physical effects induced by boundaries in dS spacetime we have considered planar coordinates having flat spatial sections. These coordinates only cover half of dS spacetime. But this does not cause any problem from the cosmological point of view. From this perspective the background geometry under consideration is just a special case of the larger class of spatially flat Friedmann-Robertson-Walker-type models, which are relevant to inflationary cosmology. Moreover, the fact that planar coordinates cover a part of dS spacetime does not cause problems in the quantization of fields because $t=$ const defines a Cauchy surface and the information from any part of the dS manifold enters the submanifold covered by these coordinates as an initial condition (for a recent discussion see [22]). The full dS spacetime is covered by global coordinates with spherical spatial sections. An important point is that the Bunch-Davies state defines the vacuum states in both planar and global coordinates. As a result, in global coordinates, the two-point functions and the VEVs for the region between the boundaries are obtained from the corresponding expressions in planar coordinates by using the standard transformation formulae for the components of tensors. Note that in the problem under consideration the planar coordinates are the most appropriate ones because the boundaries are coordinate surfaces. The latter is not the case in global coordinates and the equation of the boundaries in these coordinates is complicated.

\section{Conclusion}

We have considered the Casimir effect for the electromagnetic field in the geometry of two planar conducting boundaries on background of $(D+1)$-dimensional dS spacetime. For the Bunch-Davies 
vacuum state the two-point function for the electromagnetic field tensor is evaluated in the region between the plates. This function is expressed in terms of the boundary-free function by the formula (10) and the latter is given by (12). We consider a free field theory and all the information on the properties of the vacuum state is encoded in the two-point functions. As important local characteristics of the vacuum state, we evaluate the mean field squared and the VEV of the energymomentum tensor. These VEVs are decomposed into the boundary-free and plate-induced parts. For points away from the boundaries, the renormalization is required for the boundary-free parts only. For $D=3$ the electromagnetic field is conformally invariant and the plate-induced VEVs are expressed in terms of the corresponding quantities for conducting plates in Minkowski spacetime by standard conformal relations (see (23) and (38)).

In the region between the plates, the boundary-induced part in the VEV of the electric field squared is given by the expression (21) where the function $G_{D}(z)$ is expressed in terms of the hypergeometric function by (13). For the proper separation between the plates smaller than the $\mathrm{dS}$ curvature radius, the leading term in the electric field squared is given by (26) and this term coincides with the corresponding VEV for the plates in Minkowski spacetime with the separation $\alpha L / \eta$. In this limit the mean electric field squared is positive. In the opposite limit of large separations, assuming that $z^{D}, L-z^{D} \gg \eta$, the leading terms in the asymptotic expansion are given by (27) for $D>4$ and by (28) for $D<4$. For $D=4$ one has the asymptotic (29). At large separations, with dependence of $D$ and $z^{D} / L$, the boundary-induced VEV in the electric field squared can be either positive or negative. In the Minkowski bulk this VEV is always positive.

For the boundary-induced part in the VEV of the diagonal components of the energy-momentum tensor we have derived the expression (32) with the functions (33) and (34). In addition, for $D \neq 3$, the vacuum energy-momentum tensor has a nonzero off-diagonal component (36) that describes the energy flux along the direction normal to the plates. The off-diagonal component is antisymmetric with respect to the plane $z^{D}=L / 2$ and, hence, it vanishes at $z^{D}=L / 2$. In the region between the plates, the flux is positive for $z^{D}<L / 2$ and negative for $z^{D}>L / 2$. At small separations, the VEVs of the diagonal components of the energy-momentum tensor, to the leading order, coincide with the corresponding results for the plates in Minkowski spacetime with the separation $\alpha L / \eta$, whereas the leading term in the asymptotic expansion of the off-diagonal component is given by (44). In the limit when the separation between the plates is large compared to the dS curvature radius and assuming that $z^{D}, L-z^{D} \gg \eta$, for $D \geqslant 4$ the terms in the asymptotic expansions are given by the expressions (45) and (47).

The interaction part of the Casimir pressure on the plates is given by the expression (48) and the corresponding forces are attractive. At small separations, to the leading order we obtain the Casimir force for the geometry of two parallel plates in Minkowski spacetime with the separation $\alpha L / \eta$. For large separations and for $D \geqslant 4$, the leading term in the corresponding asymptotic expansion is given by (51). At large separations the force decays as the inverse forth power of the proper separation for all values of $D \geqslant 3$. This is in contrast with the case of the plates in Minkowski bulk where the force decays inversely proportional to the $(D+1)$ th power of the separation. Hence, for $D \geqslant 4$, in dS spacetime the decay of the Casimir forces at large separations between the plates is weaker than that for the plates in Minkowski bulk.

In the discussion above we have imposed the boundary condition which is a generalization of the perfectly conducting boundary condition for higher-dimensional models. Instead, we could impose the boundary condition $n^{\mu} F_{\mu \nu}=0$ that is used in MIT bag models of hadrons for the confinement of the color. The corresponding modes are obtained from (2) with the replacement $\sin \left(k_{D} z^{D}\right) \rightarrow$ $\cos \left(k_{D} z^{D}\right)$ and from (3) with the replacement $\cos \left(k_{D} z^{D}\right) \rightarrow \sin \left(k_{D} z^{D}\right)$. The further evaluation of the two-point functions and VEVs is similar to that we have described above. The corresponding results for the both types of boundary conditions may have applications in braneworlds on dS bulk with reflecting branes. Bearing in mind further applications in Klauza-Klein-type models with compact extra dimensions, it would be interesting to generalize the expressions for the Casimir 
densities in the case of a locally dS spacetime a part of spatial coordinates of which are compactified. The comparison of the Casimir force with the corresponding expression for the geometry in the absence of compact dimensions would give constraints on the geometry of a compact subspace (for a discussion in AdS bulk see [16]).

\section{Acknowledgments}

A. A. S. and H. A. N. were supported by the State Committee of Science Ministry of Education and Science RA, within the frame of Grant No. SCS 13-1C040.

\section{References}

[1] A. D. Linde, Particle Physics and Inflationary Cosmology (Harwood Academic Publishers, Switzerland, 1990).

[2] N. D. Birrell and P. C.W. Davies, Quantum Fields in Curved Space (Cambridge University Press, Cambridge, England, 1982); V. Mukhanov and S. Winitzki, Introduction to Quantum Effects in Gravity (Cambridge University Press, Cambridge, England, 2007); L. Parker and D. Toms, Quantum Field Theory in Curved Spacetime: Quantized Fields and Gravity (Cambridge University Press, Cambridge, England, 2009); S. Hollands and R.M. Wald, "Quantum Fields in Curved Spacetime," arXiv:1401.2026.

[3] V. M. Mostepanenko and N. N. Trunov, The Casimir Effect and its Applications (Clarendon, Oxford, 1997); K. A. Milton, The Casimir Effect: Physical Manifestation of Zero-Point Energy (World Scientific, Singapore, 2002); M. Bordag, G. L. Klimchitskaya, U. Mohideen, and V. M. Mostepanenko, Advances in the Casimir Effect (Oxford University Press, Oxford, 2009); Casimir Physics, edited by D. Dalvit, P. Milonni, D. Roberts, and F. da Rosa, Lecture Notes in Physics Vol. 834 (Springer-Verlag, Berlin, 2011).

[4] H. B. G. Casimir, Proc. K. Ned. Akad. Wet. 51, 793 (1948).

[5] L. S. Brown and G. J. Maclay, Phys. Rev. 184, 1272 (1969).

[6] B. DeWitt, Phys. Rep. 19C, 295 (1975).

[7] C. A. Lütken and F. Ravndal, Phys. Rev. A 31, 2082 (1985).

[8] H. Alnes, F. Ravndal, I. K. Wehus, and K. Olaussen, Phys. Rev. D 74, 105017 (2006); H. Alnes, K. Olaussen, F. Ravndal, and I.K. Wehus, J. Phys. A 40, F315 (2007); I. Brevik and K. A. Milton, Phys. Rev. E 78, 011124 (2008).

[9] A. Edery and V. Marachevsky, J. High Energy Phys. 12 (2008) 035.

[10] A. A. Saharian and T.A. Vardanyan, Classical Quantum Gravity 26, 195004 (2009); E. Elizalde, A. A. Saharian, and T. A. Vardanyan, Phys. Rev. D 81, 124003 (2010); A. A. Saharian, Int. J. Mod. Phys. A 26, 3833 (2011); K. A. Milton and A. A. Saharian, Phys. Rev. D 85, 064005 (2012).

[11] M. R. Setare and R. Mansouri, Classical Quantum Gravity 18, 2331 (2001); M. R. Setare, Classical Quantum Gravity 18, 4823 (2001); P. Burda, JETP Lett. 93, 632 (2011). 
[12] A. A. Saharian and M. R. Setare, Phys. Lett. B 659, 367 (2008); S. Bellucci and A. A. Saharian, Phys. Rev. D 77, 124010 (2008); A. A. Saharian, Classical Quantum Gravity 25, 165012 (2008); E. R. Bezerra de Mello and A. A. Saharian, J. High Energy Phys. 12 (2008) 081; S. Bellucci, A. A. Saharian, and H. A. Nersisyan, Phys. Rev. D 88, 024028 (2013).

[13] S. Bellucci, A. A. Saharian, and A. H. Yeranyan, Phys. Rev. D 89, 105006 (2014).

[14] A. A. Saharian, A. S. Kotanjyan, and H. A. Nersisyan, Phys. Lett. B 728, 141 (2014).

[15] S. Bellucci and A. A. Saharian, Phys. Rev. D 88, 064034 (2013).

[16] L. P. Teo, J. High Energy Phys. 10 (2010) 019.

[17] J. Ambjørn and S. Wolfram, Ann. Phys. (N.Y.) 147, 1 (1983).

[18] T. S. Bunch and P. C. W. Davies, J. Phys. A 11, 1315 (1978).

[19] B. Allen and T. Jacobson, Commun. Math. Phys. 103, 669 (1986); J.-P. Gazeau, M. V. Takook, J. Math. Phys. 41, 5920 (2000); T. Garidi, J.-P. Gazeau, S. Rouhani, M. V. Takook, J. Math. Phys. 49, 032501 (2008).

[20] A. Youssef, Phys. Rev. Lett. 107, 021101 (2011).

[21] Handbook of Mathematical Functions, edited by M. Abramowitz and I. A. Stegun (Dover, New York, 1972).

[22] H. Degueldre and R. P. Woodard, Eur. Phys. J. C 73, 2457 (2013). 